\title{
Bilateral Renal Involvement From a Primary Brain Tumor
}

\author{
Adam Alleemudder ${ }^{\mathrm{a}}$, Rajiv Pillai ${ }^{\mathrm{a}, \mathrm{b}}$
}

\begin{abstract}
We describe only the third case of bilateral renal metastases from a primary brain hemangiopericytoma (HPC) in a 58-year-old male. These rare malignant tumors were first described in 1942 and originate from pericytes located around capillaries. They can therefore originate from anywhere in the body. They may be confused with primary renal cell carcinoma when they metastasize to the kidneys. Surgical management remains the mainstay of treatment for renal involvement.
\end{abstract}

Keywords: Hemangiopericytoma; Renal involvement; Brain tumor

\section{Introduction}

Hemangiopericytomas (HPCs) were first described in 1942 and are tumors with rapid growth and high malignant potential that originate from pericytes that surround capillaries. They can potentially originate from anywhere in the body but common sites include the head and neck, pelvis and the extremities. Metastasis to the kidneys is a rare event, which is often a carcinoma that originates more commonly from the lung or colon. Renal metastasis from a primary brain HPC, especially when bilateral, is even rarer. In most cases, the patient is asymptomatic until an advanced stage of the disease is reached when there may be hematuria, loin pain or even hypoglycemia from excessive tumor metabolism. The majority of cases therefore are detected through surveillance cross-sectional imaging of the original tumor and in the majority, surgical excision remains the mainstay of management. In this article, we describe only the third ever documented case of bilateral renal involvement from a primary brain $\mathrm{HPC}$ and review the literature to describe the salient features of HPC and its management.

\section{Case Report}

We present the rare case of a 58-year-old Caucasian male who

Manuscript accepted for publication February 24, 2016

${ }^{a}$ Colchester General Hospital, Turner Road, Colchester, Essex CO4 5JL, UK ${ }^{\mathrm{b} C o r r e s p o n d i n g ~ A u t h o r: ~ C o l c h e s t e r ~ G e n e r a l ~ H o s p i t a l, ~ T u r n e r ~ R o a d, ~ C o l c h e s-~}$ ter, Essex CO4 5JL, UK. Email: adamalleemudder@doctors.org.uk developed bilateral metastatic renal hemangiopericytomas 14 years after undergoing resection of the primary from the posterior cranial fossa. During this period, a small cranial recurrence was further resected and a small solitary lung metastasis confirmed on biopsy was identified. It was during surveillance for the lung lesion that a new $2.4 \mathrm{~cm}$ right upper pole and a $2 \mathrm{~cm}$ left lower pole renal masses were detected on CT scanning which raised suspicion of secondary metastases in view of the history (Fig. 1). There was an initial decision to keep these lesions under surveillance because of their size but in view of the patient's symptoms and heightened anxiety, a left partial nephrectomy was undertaken in the first instance with histology subsequently again confirming HPC (Fig. 2). At the present time, it remains the intention to keep both the lung and right renal lesions under surveillance until such time there are any concerns.

\section{Discussion}

To our knowledge, this is only the third described case of bilateral renal metastases from a primary brain $\operatorname{HPC}[1,2]$. In contrast to the two other studies, which describe a radical nephrectomy for the metastatic lesion, a nephron sparring approach was undertaken in our case.

Metastasis to the kidneys is generally a rare event which

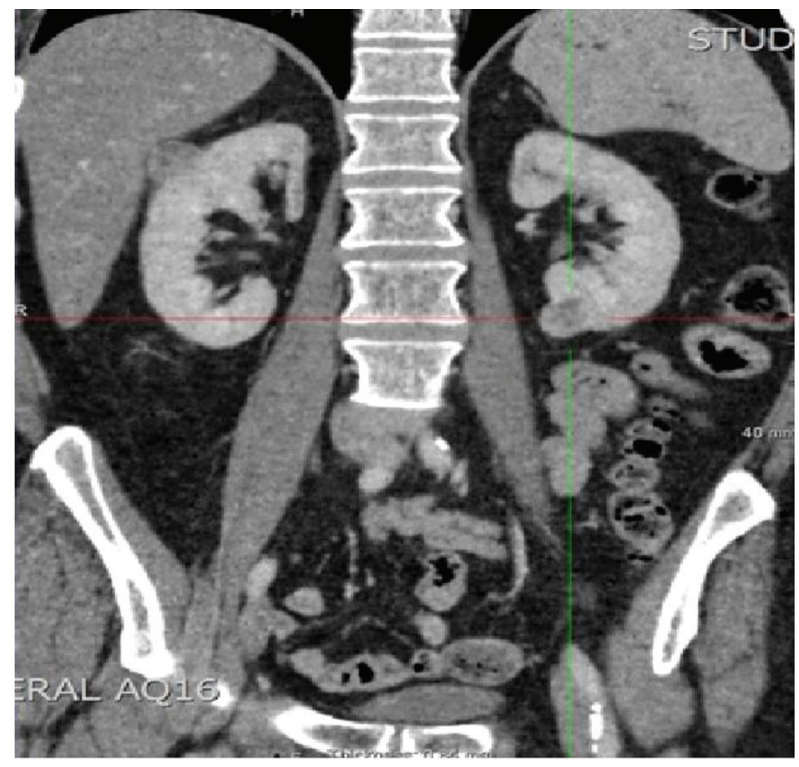

Figure 1. CT showing bilateral renal masses. 




Figure 2. Microscopic appearance of HPC showing densely packed population of spindle or oval shaped cells with elongated nuclei separated by slit-like vascular spaces.

could be confused with the development of a primary renal cell carcinoma. In the vast majority of cases, the lesions tend to be carcinomas and large autopsy studies have previously confirmed an incidence rate ranging between $7.2 \%$ and $12.6 \%$ $[3,4]$. There appears to be a higher incidence in males and Caucasians, with secondary renal metastases occurring in the fifth decade [5]. The two most common sites for the originating primary are the lungs and colon. Other sites with lesser tendency include breast, skin and thyroid [6]. Involvement of the kidneys may be solitary, multifocal, ipsilateral or bilateral [7]. The time taken to the development of renal metastases also varies with one study reporting a mean time of 26 months whilst another was 63 months [5, 8].

The development of metastases from a primary brain tumor is even rarer. Our case developed from a primary brain HPC which tends to be unilateral only [9]. HPCs originate from pericytes that surround capillaries and regulate blood flow and permeability. They can therefore develop from a multitude of sites around the body, including the kidney, but predominantly originate from the extremities, head and neck, pelvis and extremities [10]. Stout and Murray in 1942 became the first authors to define HPC as a distinct vascular neoplasm whilst renal HPCs were first noted by Black and Heinemann several years later $[11,12]$. They are highly vascularized soft tissue tumors surrounded by a capsule or pseudocapsule often with high rapid growth and malignant potential. Microscopically, the tumor comprises of a densely packed population of spindle or oval shaped cells with elongated nuclei, often separated by slit-like vascular spaces (Fig. 2). The cells lack pleomorphism or hyperchromasia and have positive immunostaining for CD34 and vimentin.

The only two case reports of bilateral renal involvement from a brain HPC in the literature both occurred in males and 10 years after the initial diagnosis; Heppe originally described the first case occurring in a 41-year-old patient presenting with flank pain and hematuria and who eventually underwent a right radical nephrectomy and left heminephrectomy [1]. Hammontree et al subsequently reported a simi- lar case in a 37-year-old patient who had a left nephrectomy shortly followed by a $4 \mathrm{~mm}$ recurrence in the right kidney which is presumed to have been kept under surveillance initially [2].

In keeping with the incidental detection rate of RCC on imaging performed for other reasons, over half of patients who develop renal metastases are asymptomatic. Surveillance scans for the original primary tumor often lead to their detection. It is often the case that presentation with symptoms indicates advanced or aggressive disease. In one study, two of the 13 patients with an original lung tumor presented quite early on with hematuria [5]. In another retrospective study, where 151 patients with a primary non-renal malignancy with renal metastases were studied, the commonest symptoms were abdominal or flank pain, followed by hematuria, weight loss, night sweats and fever, with $56.7 \%$ being asymptomatic [6]. In particular to renal HPC, hypoglycemia from excessive tumor metabolism and hypertension from the release of mediators may also occur [10].

Detection of secondary renal lesions is often through the use of CT scans. It may be difficult, however, to distinguish a renal primary from secondary renal lesions due to a lack of clearly definable pathognomic criteria [13]. Inter-observer discordance also plays a key role. This is highlighted by Zhou et al, who noted radiologists were more likely to report a renal mass as a primary rather than a secondary although concordance between radiologists and clinician assessments seemed to correlate in $54 \%$ of the cases [6]. Metastatic lesions in general though tend to be multifocal with low attenuation [14]. There appears to be a lack of correlation for metastatic disease whether the lesions are exophytic or endophytic [15]. When doubt exists regarding the diagnosis, needle biopsy of the suspect lesion may be undertaken, but due to histological similarities, there may be difficulty in distinguishing between HPC and other soft tissue tumors such as synovial sarcomas and fibrous histiocytomas [10].

The development of disseminated disease is a poor prognostic indicator and systemic chemotherapy targeted to the original tumor is often required depending on the patient's fitness. It is often quite rare that the kidneys are the sole destination for metastases from a known primary. If so, it is usually not long before other sites quickly become involved. The single most important prognostic indicator is the presence or absence of metastases [2]. Surgical resection of solitary metastases may be a viable option in well-selected patients. The case series from Adamy et al had 13 patients who underwent nephrectomy for solitary kidney metastases from a variety of primary tumors. Four patients succumbed to disease progression but eight patients were alive 30 months after surgery, with four of these having no evidence of active disease. The authors concluded that nephrectomy could provide a survival benefit in well-selected group of patients [5]. Zhou et al demonstrated that of the 151 patients, the median overall survival from renal metastases was almost double in those who underwent surgery. Therefore, complete or nephron sparing surgery, as described in our case, seems to a plausible option in those with solitary secondary renal lesions. However, the role of cytoreductive nephrectomy in patients with widespread disseminated disease is yet to be established. 


\section{References}

1. Heppe RK, Donohue RE, Clark JE. Bilateral renal hemangiopericytoma. Urology. 1991;38(3):249-253.

2. Hammontree LN, Whitehead K, Markert JM. Bilateral metastatic renal hemangiopericytoma ten years after primary intracranial lesion. Int Braz J Urol. 2006;32(3):306307.

3. Abrams HL, Spiro R, Goldstein N. Metastases in carcinoma; analysis of 1000 autopsied cases. Cancer. 1950;3(1):74-85.

4. Bracken RB, Chica G, Johnson DE, Luna M. Secondary renal neoplasms: an autopsy study. South Med J. 1979;72(7):806-807.

5. Adamy A, Von Bodman C, Ghoneim T, Favaretto RL, Bernstein M, Russo P. Solitary, isolated metastatic disease to the kidney: Memorial Sloan-Kettering Cancer Center experience. BJU Int. 2011;108(3):338-342.

6. Zhou C, Urbauer DL, Fellman BM, Tamboli P, Zhang M, Matin SF, Wood CG, et al. Metastases to the kidney: a comprehensive analysis of 151 patients from a tertiary referral centre. BJU Int. 2015.

7. Bhatt GM, Bernardino ME, Graham SD, Jr. CT diagnosis of renal metastases. J Comput Assist Tomogr.
1983;7(6):1032-1034.

8. Choyke PL, White EM, Zeman RK, Jaffe MH, Clark LR. Renal metastases: clinicopathologic and radiologic correlation. Radiology. 1987;162(2):359-363.

9. Hietala SO, Wahlqvist L. Metastatic tumors to the kidney. A postmortem, radiologic and clinical investigation. Acta Radiol Diagn (Stockh). 1982;23(6):585-591.

10. Brescia A, Pinto F, Gardi M, Maria Vecchio F, Bassi PF. Renal hemangiopericytoma: case report and review of the literature. Urology. 2008;71(4):755 e759-712.

11. Stout AP, Murray MR. Hemangiopericytoma: A Vascular Tumor Featuring Zimmermann's Pericytes. Ann Surg. 1942;116(1):26-33.

12. Black HR, Heinemann S. Hemangiopericytoma: report of a case involving the kidney. J Urol. 1955;74(1):42-46.

13. Pollack HM, Banner MP, Amendola MA. Other malignant neoplasms of the renal parenchyma. Semin Roentgenol. 1987;22(4):260-274.

14. Bailey JE, Roubidoux MA, Dunnick NR. Secondary renal neoplasms. Abdom Imaging. 1998;23(3):266-274.

15. Honda H, Coffman CE, Berbaum KS, Barloon TJ, Masuda K. CT analysis of metastatic neoplasms of the kidney. Comparison with primary renal cell carcinoma. Acta Radiol. 1992;33(1):39-44. 